AperTO - Archivio Istituzionale Open Access dell'Università di Torino

Risk reducing surgery and treatment of menopausal symptoms in BRCA mutation carriers (and other risk women)

This is a pre print version of the following article:

Original Citation:

Availability:

This version is available http://hdl.handle.net/2318/1698768

since 2019-04-17T13:30:44Z

Publisher:

Springer

Terms of use:

Open Access

Anyone can freely access the full text of works made available as "Open Access". Works made available under a Creative Commons license can be used according to the terms and conditions of said license. Use of all other works requires consent of the right holder (author or publisher) if not exempted from copyright protection by the applicable law. 


\section{Risk reducing surgery and treatment of menopausal symptoms in BRCA mutation carriers (and other risk women)}

Piero Sismondi, Marta D’Alonzo, Paola Modaffari, Viola Liberale, Valentina Elisabetta Bounous, Andrea Villasco, Nicoletta Biglia Department of Obstetrics and Gynaecology, University of Turin, "Umberto I Hospital”, Turin, Italy

\section{Introduction and Risk Assessment}

In developed countries Breast Cancer (BC) occurs in one out of eight women during her lifetime, estimating the life expectancy of 85 years. About $10 \%$ of $\mathrm{BC}$ are associated with genetic risk factors, essentially mutations of BRCA1 and BRCA2, however but the majority of BC are sporadic. Risk factors for $\mathrm{BC}$ are primarily related to age and to estrogen exposure (early menarche, late menopause, nulliparity, use of exogenous hormones), in addition high risk population also include women with atypical hyperplasia, and patients with ductal or lobular carcinoma in situ.

The lifetime cumulative risk of breast cancer for women with BRCA1 or BRCA2 mutations is very high ranging from 45 to $65 \%$, this population has an elevated ovarian cancer risk as well ${ }^{1}$. The ovarian cancer estimated lifetime risks is $36-46 \%$ and $10-27 \%$ in $B R C A 1$ and $B R C A 2$ mutation carriers, respectively. Therefore for these women a close surveillance is suggested as well as medical and surgical options, including chemoprevention, bilateral salpingo-oophorectomy and mastectomy.

Outside of genetic risk factors, for general population, it's important to identify women at high risk of breast cancer: patients with previous thoracic RT $<30$ y of age, women diagnosed with lobular carcinoma in situ, women with an estimated 5 -year breast cancer risk $\geq 1.7 \%{ }^{2}$. Several mathematical models to estimate this risk have been proposed, currently the most used is the National Cancer Institute (NCI) Breast Cancer Risk Assessment Tool which is a modified version of the Gail model that consider age, race, age at first pregnancy, family history and history of atypical hyperplasia; individuals with a 5 -year risk of $1.66 \%$ or greater are considered at risk ${ }^{3}$. All the models have a limited 
reliability and, as a matter of fact, up to $60 \%$ of $\mathrm{BC}$ occurs in women with no known risk factors; the available models do not include some risk factors such as obesity, diet, mammographic density and use of HRT. A higher accuracy could be obtained by incorporating information on genotypes as well.

A recent report published on the new England journal of medicine ${ }^{4}$ underlines the importance of atypical hyperplasia as an independent factor for the inclusion of patients in chemoprevention programs. Atypical hyperplasia is a high-risk benign lesion that is found in approximately $10 \%$ of biopsies with benign findings. In studies with long-term follow-up, atypical hyperplasia has been shown to confer a relative risk for future breast cancer of 4 . Another large cohort study at the Mayo Clinic $^{5}$ published on 2014, confirms the cumulative high risk of breast cancer among women with atypical hyperplasia. Indeed, 25 years after a biopsy showing atypical hyperplasia, breast cancer (either in situ or invasive) developed in $30 \%$ of the women, with greater numbers of foci associated with a higher risk. Hartman and co. conclude suggesting that absolute risk data about women diagnosed with atypical hyperplasia should be used instead of models to describe breast-cancer risk in this population. Guidelines for high-risk women should be updated to include women with atypical hyperplasia. Analyses of data from the subgroup of women with atypical hyperplasia were performed in four of the placebo-controlled trials (NSABP P-1, MAP.3, IBIS-I, and IBIS-II). A total of 2009 women with atypical hyperplasia were randomly assigned to receive an active agent or placebo in these trials. Relative-risk reductions in the atypical hyperplasia subgroup ranged from 41 to $79 \%$, which suggested an even greater benefit than in the total population treated with active agent in these trials.

Women with a life expectancy of $\geq 10$ years and no diagnosis or history of breast cancer who are considered to be at increased risk for breast cancer based on any of the above-mentioned assessments, should receive individualized counseling to decrease breast cancer risk. Strategies for prevention of breast cancer include: lifestyle factors (avoidance of obesity, maintaining physical activity, moderation in alcohol intake), chemoprevention therapy with risk-reduction agents and risk-reduction surgery ${ }^{2}$. 


\section{Risk reduction mastectomy (RRM)}

Retrospective analyses with median follow-up periods of 13 to 14 years have indicated that bilateral risk-reducing mastectomy decreased the risk of developing breast cancer by approximately $90 \%$ in moderate- and high-risk women and in known $B R C A 1 / 2$ mutation carriers ${ }^{6}$. Further results from smaller prospective studies with shorter follow-up support the conclusion that RRM provides a high degree of protection against breast cancer in women with a $B R C A 1 / 2$ mutation. A recent meta-analysis including 2635 patients, demonstrated a significant risk reduction of breast cancer incidence in BRCA1 and BRCA2 mutation carriers receiving RRM (HR 0.07; $95 \%$ CI 0.01-0.44; $p=0.004$ ).

In the 2007 guidelines issued by the Society of Surgical Oncology, indications for bilateral prophylactic mastectomies in Patients without a Cancer Diagnosis included: BRCA mutations or other genetic susceptibility genes, strong family history with no demonstrable mutation, histological risk factors (including atypical ductal or lobular hyperplasia, or lobular carcinoma in situ confirmed on biopsy); a further indication was a difficult surveillance ${ }^{7}$. The position Statement states that rarely, bilateral prophylactic mastectomies may be warranted for an exceptional patient with no family history or high-risk histology such as patient with extremely dense fibronodular tissue that is difficult to evaluate with standard breast imaging, several prior breast biopsies for clinical and/or mammographic abnormalities, and strong concern about breast cancer risk. In the same guidelines, potential indications for prophylactic contralateral mastectomy in patients with a current or previous diagnosis of breast cancer were: risk reduction (see indications as listed above), difficult surveillance (patients with clinically and mammographically dense breast tissue or diffuse indeterminate microcalcifications in the contralateral breast), or reconstructive issues (symmetry/balance).

The 2017 NCCN Breast Cancer Risk Reduction Panel supports the use of RRM for carefully selected women at high risk for breast cancer who desire this intervention, exclusively considering BRCA1/2 or other genetic mutations and previous history of LCIS'. There are no data regarding RRM in women with prior mantle radiation exposure. As regard Atypical hyperplasia, the Society of Surgical 
Oncology recognizes it as a possible but not routine indication for bilateral prophylactic mastectomy ${ }^{7}$. The recent report published on the new England Journal of Medicine on Atypical hyperplasia and possible surgical Risk Reduction Interventions, concludes that, in current practice, with minimal data available on this topic and with chemopreventive agents for risk reduction available, atypical hyperplasia is generally not an indication for prophylactic mastectomy ${ }^{32}$.

Women considering RRM should first have appropriate multidisciplinary consultations, a clinical breast examination and bilateral mammogram if not performed within the past 6 months. If results are normal, women who choose RRM may undergo the procedure with or without immediate breast reconstruction. Axillary node assessment has limited utility at the time of RRM. Women undergoing RRM do not require an axillary lymph node biopsy unless breast cancer is identified on pathologic evaluation of the mastectomy specimen. Following RRM, for monitoring breast health, women should continue with annual exams of the chest and the reconstructed breast because there is still a small residual risk of developing breast cancer. Mammograms are not recommended in this situation ${ }^{2}$.

\section{Bilateral risk-reducing salpingo-oophorectomy (RRSO)}

The absence of reliable methods of early detection and the poor prognosis associated with advanced ovarian cancer have lent support for the performance of bilateral risk-reducing salpingo-oophorectomy (RRSO) that decreases the risk of developing ovarian and fallopian cancer by $85-95 \%$ in BRCA1/2 mutation carriers when performed before age $50^{8}$. RRSO is also reported to reduce by approximately $50 \%$ the risk for breast cancer in BRCA mutation carriers when performed in premenopausal age. The results of several studies suggest that RRSO may be associated with a greater reduction in breast cancer risk for BRCA1 mutation carriers compared with BRCA2 mutation carriers ${ }^{9}{ }^{10}$.

The decreased hormonal exposure following surgical removal of the ovaries is the basis for the reductions in breast cancer risk after RRSO; results from Eisen et al suggest the reductions in breast cancer risk is greater when the surgery is performed in younger women: (OR 0.41 (95\% CI, 0.25-0.68) for RRSO performed at age 40 years or younger versus odds ratio 0.47 (95\% CI, 0.28-0.80) for RRSP 
performed in carriers aged 41-50 years). Non significant risk reduction of breast cancer was found for women aged 51 years or older ${ }^{10}$. Data are limited about the optimal age for RRSO. Considering that the mean age at diagnosis of ovarian cancer is 50.8 year for BRCA1/2 mutation carriers ${ }^{11}$, current guidelines for ovarian cancer risk management recommended bilateral salpingo-oophorectomy at the completion of childbearing or by age 35 to $40^{2}$. Considering the slight advance of the diagnosis in BRCA 1 carriers than in BRCA 2, in the first group of patients, the RRSO could be proposed as soon as possible after the completion of childbearing, delaying the surgery after 40 years only after careful consideration of risks and benefits. In BRCA2 mutated patients, instead, the action may be brought between 40 and 50 years considering the progressive reduction of the protective effect against breast cancer $^{12}$.

Following prophylactic salpingo-oophorectomy a $1-4.3 \%$ residual risk for a primary peritoneal carcinoma in BRCA1 and BRCA2 mutation carriers still exists ${ }^{13}$. It cannot be excluded that in some cases peritoneal carcinoma foci are actually metastases of sub-clinical disease that was present at the time of surgery (occult carcinomas), so that undiagnosed cancers at the time of surgery will be considered primary peritoneal cancer when they become clinically apparent. Possibly fewer peritoneal cancers will be diagnosed after salpingo-oophorectomy if the comprehensive pathology review of the specimens is performed on all patients ${ }^{14}$. Fisch et al in paper based on a series of 159 female BRCA1 or BRCA2 carriers who underwent prophylactic oophorectomy, showed that $2-10 \%$ of BRCA1/2 carriers who undergo prophylactic salpingo-oophorectomy will be found to have occult carcinomas if the ovaries and the tubes are rigorously examined. No cancers were detected among women who had the operation at age 39 or younger ${ }^{15}$. The conclusion is that a rigorous operative and pathologic protocol for RRSO increases the detection rate of occult ovarian malignancy in BRCA mutation carriers, influencing the postoperative management whit additional staging, chemotherapy, and followup in affected women. Also the peritoneal lavage cytology can detect occult carcinoma at the time of $\mathrm{RRSO}^{16}$ and should always be performed ${ }^{2}$. The additional benefit of concurrent hysterectomy is not clear. Even if careful ligation of the fallopian tube at the uterine origin is performed, a small portion of 
interstitial fallopian tube in the cornua of the uterus is left in situ if hysterectomy is not performed, however, in the largest study on fallopian tube cancer to date, $92 \%$ of cancers originated in the distal or midportion of the tube. The concurrent hysterectomy, can simplify HRT allowing estrogen only supplementation and can reduce the endometrial cancer risk associated with Tamoxifen treatment for a previous breast cancer ${ }^{12}$. However the risk and benefits of concomitant hysterectomy should be discussed with each individual woman. As the majority of BRCA-associated ovarian cancers appear to originate in the fallopian tube, some Authors propose prophylactic salpingectomy; data on short- and long-term outcomes of this prophylactic surgery is limited and there are no studies directly comparing prophylactic salpingectomy with bilateral salpingo-oophorectomy for BRCA mutated women. A clinical trial led by Leblanc et al is currently recruiting young BRCA mutation carriers for radical fimbriectomy (NCT016808074), but it is not expected to be complete until $2019^{17}$. A two-stage procedure has been proposed: bilateral salpingectomy with delayed oophorectomy to prevent the adverse consequences of premature menopause. Know et al developed a Markov Monte Carlo simulation model to compare three strategies for risk reduction in women with BRCA mutations: bilateral salpingo-oophorectomy; bilateral salpingectomy; bilateral salpingectomy with delayed oophorectomy. The model estimates the number of future breast and ovarian cancers and cardiovascular deaths attributed to premature menopause with each strategy: bilateral salpingooophorectomy offers the greatest risk reduction for breast and ovarian cancer, but when considering quality-adjusted life expectancy, bilateral salpingectomy with delayed oophorectomy is a costeffective strategy and may be an acceptable alternative for those unwilling to undergo immediate bilateral salpingo-oophorectomy ${ }^{18}$. Finally some authors proposed the tubal ligation as a feasible option to reduce the risk of ovarian cancer in women with BRCA mutations who have completed childbearing. Four main mechanisms are invoked: a screening effect, the alteration of ovarian function, a mechanical barrier to the ascent of endometrial or proximal fallopian tube cells into the peritoneal cavity and the prevention of retrograde transport of carcinogenic substances from the vagina. A metaanalysis of 13 studies shows a reduced risk of epithelial ovarian cancer by $34 \%$. The protective effect 
of tubal ligation was confirmed even in a subgroup of women 10-14 years after the procedure. The risk reduction was confirmed for the endometrioid and serous cancers but not for mucinous ${ }^{19}$.

\section{Treatment of menopausal symptoms in BRCA mutation carriers}

The absence of reliable methods of early detection and the poor prognosis associated with advanced ovarian cancer have lent support for the performance of bilateral risk reduction RRSO, however there are many concerns and worries about the resulting premature menopause.

The most common symptoms are: vasomotor symptoms (hot flashes, night sweats, palpitations), vaginal dryness, sexual dysfunction, cognitive dysfunction, poor sleep and tiredness. Women who were premenopausal at the time of surgery experienced a worsening of hot flashes, night sweats and sweating and a decline in sexual function 1 year after surgery. Menopausal disorders are more severe if RRSO is performed in pre menopausal women than in post menopausal women ${ }^{20}$.

Premenopausal oophorectomy is also associated with an increased risk of osteopenia, osteoporosis and fracture. Following oophorectomy, there is an increased prevalence of osteoporosis within three to six years of surgery, the loss of trabecular bone has been reported to be as high as $20 \%$ during the first 18 months following surgery. Several recent cross-sectional studies have examined bone health after salpingo-oophorectomy in women with a BRCA mutation. Cohen et al. published a study of 226 BRCA carriers after salpingo-oophorectomy ${ }^{21}$ : among women who underwent surgery before the age of 50 years, high rates of osteopenia (62\%) and osteoporosis $(9 \%)$ were reported. The 2010 Canadian guidelines for diagnosis and management of osteoporosis proposes bone density measurement with DXA at the time of salpingo-oophorectomy and again 1-2 years after surgery; the timing of further bone density measurements should be individualized based on the results of these two measurements; to prevent osteoporosis, a recommended daily intake of $1500 \mathrm{mg}$ calcium from dietary and supplemental sources and supplementation with $800 \mathrm{IU} /$ day of vitamin D daily is suggested. In women with premature menopause Hormone Replacement Therapy (HRT), if not contraindicated, is advised to preserve bone mineral density, however the duration of use is not clear ${ }^{2}$. 
Bilateral oophorectomy has also been shown to be a risk factor for coronary heart disease. A positive association between bilateral oophorectomy and increased risk of cardiovascular disease has been observed in a number of observational studies, including the Nurse's Health Study (rate ratio 2.2) and the Mayo Clinic Cohort of Oophorectomy and Aging (HR 1.4). A recent study ${ }^{22}$ links BRCA1 gene function with cardiovascular function, ( RCA1 gene products work to prevent DNA damage). The loss of BRCA1 in cardiomyocytes may results in adverse cardiac remodeling, poor ventricular function and increased mortality in response to ischemic or genotoxic stress. Therefore BRCA1-mutated women may be at an increased risk for cardiovascular disease. Even if there are no clinical studies to date to evaluate this issue. Also in this context HRT may mitigate the increase in cardiovascular risk associated with surgical menopause.

Several studies show that short-term HRT use does not negate the protective effect of RRSO on subsequent breast cancer risk in BRCA1/2 mutation carriers ${ }^{23}$. In a matched case-control study of 472 postmenopausal women with a BRCA1 mutation, Eisen et al. examined whether or not the use of HRT was associated with subsequent risk of breast cancer ${ }^{24}$. The adjusted OR for breast cancer associated with ever use of HRT compared with never use was $0.58(95 \% \mathrm{CI} 0.35-0.96 ; \mathrm{P}=.03)$, they concluded that HRT use was not associated with increased risk of breast cancer; indeed, in BRCA1 mutated women, it was associated with a decreased risk. In a recent review Marchetti and $\mathrm{al}^{25}$ conclude that HRT generally reduces symptoms related to surgical menopause, short-term HRT seems to improve quality of life and does not seem to have an adverse effect on oncologic outcomes in BRCA1 and BRCA2 mutation carriers without a personal history of breast cancer. The 2013 NICE guidelines recommends HRT for women with no personal history of breast cancer, including BRCA1 or BRCA2 mutation carriers, having had bilateral salpingo-oophorectomy before their natural menopause. They should take combined HRT or Estrogens only depending on having the uterus or not, until the time they would have expected natural menopause.

Estrogen therapy, however, is contraindicated for breast cancer survivors ${ }^{26}$. The Stockholm trial ${ }^{27}$ was prematurely stopped in 2003 when the parallel HABITS trial ${ }^{28}$ reported a higher recurrence rate in 
breast cancer patients who received systemic estrogens compared to women treated with placebo. At 4 years of follow-up, the HABITS study still found an increased risk of recurrence ${ }^{29}$ whereas a recent updated analysis of the data from the Stockholm trial at 10.8 years of follow-up did not show any excess of recurrence risk $^{30}$. An alternative compound to conventional estrogen/progestogen treatment, tibolone, was tested versus placebo in the LIBERATE trial, showing a significant superiority to placebo in reducing vasomotor symptoms and improving sleep quality, sexual behaviour, mood and attraction, but unfortunately also a significant increase in the recurrence rate ${ }^{31}$.

Because of this concern, research efforts have focused on non-hormonal drugs to alleviate climacteric symptoms. Several substances have been tested: some of them give similar results to placebo, that in itself provides a response in around $20-30 \%$ of women, others have shown promising results.

\section{Conclusions}

Women with a life expectancy $\geq 10$ years and no diagnosis/history of breast cancer who are considered to be at increased risk for breast cancer, should receive counseling to decrease breast cancer risk, considering: lifestyle factors, therapy with risk-reduction agents, risk-reduction surgery (in BRCA1/2 mutation carriers).

Bilateral risk reducing mastectomy decreases the risk of developing breast cancer by at least $90 \%$; it should be proposed to carefully selected women at high risk for breast cancer considering BRCA1/2 or other genetic mutations and previous history of LCIS. In current practice, atypical hyperplasia is not an indication for prophylactic mastectomy.

Bilateral risk reducing salpingo-oophorectomy decreases the risk of developing ovarian and fallopian cancer by $85-95 \%$ and breast cancer by $50 \%$ in BRCA1/2 mutation carriers when performed in premenopausal age. Peritoneal washing should be performed at surgery and pathologic assessment should include fine sectioning of the ovaries and fallopian tubes. The additional benefit of concurrent hysterectomy is not clear at the time. In women with no personal history of breast cancer short-term 
HRT use does not negate the protective effect of RRSO on subsequent breast cancer risk and it should be offered until the time of expected natural menopause.

1 Antoniou A, Pharoah P, Narod S, Risch HA et al. Average risks of breast and ovarian cancer associated with BRCA1 or BRCA2 mutations detected in case Series unselected for family history: a combined analysis of 22 studies. Am J Hum Genet. 2003 May;72(5):1117-30

\section{NCCN Guidelines 1.2017 December 16,2016. Breast Cancer Risk Reduction}

3Mitchell H. Gail, Joseph P. Costantino, Validating and Improving Models for Projecting the Absolute Risk of Breast Cancer, Journal of the National Cancer Institute, Vol. 93, No. 5, March 7, 2001

4 Lynn C. Hartmann, et al. Atypical Hyperplasia of the Breast, Risk Assessment and Management Options. $N$ engl $j$ med 372;. January 1, 2015

5 Hartmann LC, Radisky DC, Frost MH, et al. Understanding the premalignant potential of atypical hyperplasia through its natural history: a longitudinal cohort study. Cancer Prev Res (Phila) 2014;7:211-7.

6 Hartmann et al. Efficacy of bilateral prophylactic mastectomy in women with a family history of breast cancer. $N$ Engl $J$ Med 1999

7 Armando E. Giuliano, Susan Boolbol, Amy Degnim, et al. Society of Surgical Oncology: Position Statement on Prophylactic Mastectomy. Approved by the Society of Surgical Oncology Executive Council, March 2007. Annals of Surgical Oncology 14(9)

8 Rebbeck, Kauff, Domchek. Meta-analysis of risk reduction estimates associated with risk-reducing salpingo oophorectomy in BRCA1 or BRCA2 mutation carriers. J Natl Cancer Inst 2009;101:80-87

9 Kauff, Domchek SM, Friebel, et al. Risk-reducing salpingooophorectomy for the prevention of BRCA1-and BRCA2associated breast and gynecologic cancer: a multicenter, prospective study. J Clin Oncol 2008;26:1331-1337

10 Eisen A, Lubinski J, Klijn J, et al. Breast cancer risk following bilateral oophorectomy in BRCA1 and BRCA2 mutation carriers: an international case-control study. J Clin Oncol 2005;23:7491-7496

11 Rebbeck, Lynch, Neuhausen et al. Prophylactic oophorectomy in carriers of BRCA1 or BRCA2 mutations. N Engl J Med. 2002 May 23;346(21):1616-22

12 RIGENIO guidelines Regione Piemonte 2007

13 Finch A, Beiner M, Lubinski J, et al. Salpingo-oophorectomy and the risk of ovarian, fallopian tube, and peritoneal cancers in women with a BRCA1 or BRCA2 Mutation. JAMA 2006;296:185-192.

14 Ofer Lavie, Gila Hornreich, Alon Ben-Arie, Gad Rennert, Yoram Cohen et al. BRCA germline mutations in Jewish women with uterine serous papillary carcinoma. Gynecologic Oncology 92 (2004) 521-524

15 Finch A, Shaw P, Rosen B et al. Clinical and pathologic findings of prophylactic salpingo-oophorectomies in 159 BRCA1 and BRCA2 carriers. Gynecologic Oncology 2006;100: 58 - 64

16 Colgan TJ, Boerner SL, Murphy J, Cole DE, Narod S, Rosen B. Peritoneal lavage cytology: an assessment of its value during prophylactic oophorectomy. Gynecol Oncol. 2002 Jun;85(3):397-403 
18 Janice S. Kwon, MD, Anna Tinker, MD, Gary Pansegrau et al. Prophylactic Salpingectomy and Delayed Oophorectomy as an Alternative for BRCA. Obstet Gynecol 2013;121:14-24

19 Cibula, Widschwendter, Ma'jek and Dusek. Tubal ligation and the risk of ovarian cancer: review and meta-analysis. Human Reproduction Update, Vol.17, No.1 pp. 55-67, 2011

20 Finch A, Metcalfe KA, Chiang JK, Elit L, McLaughlin J, Springate C et al. The impact of prophylactic salpingooophorectomy on menopausal symptoms and sexual function in women who carry a BRCA mutation. Gynecol Oncol. 2011 Apr;121(1):163-8

21 Cohen JV, Chiel L, Boghossian L, Jones M, Domchek et al. Non-cancer endpoints in BRCA1/2 carriers after riskreducing salpingo-oophorectomy. Fam Cancer. 2012 Mar;11(1):69-75

22 Shukla PC, Singh KK, Quan A et al. BRCA1 is an essential regulator of heart function and survival following myocardial infarction. Nat Commun. 2011 Dec 20;2:593

23 Rebbeck TR, Friebel T, Wagner T et al. Effect of Short-Term Hormone Replacement Therapy on Breast Cancer Risk Reduction After Bilateral Prophylactic Oophorectomy in BRCA1 and BRCA2 Mutation Carriers: The PROSE Study Group. J Clin Oncol 2005; 23:7804-7810

24 Eisen A, Lubinski J, Gronwald J, Moller P, Lynch HT et al. Hormone therapy and the risk of breast cancer in BRCA1 mutation carriers. . J Natl Cancer Inst. 2008 Oct 1;100(19):1361-7

25 Marchetti C, Iadarola R, Palaia I, di Donato V, Perniola G, Muzii L, Panici PB. Hormone therapy in oophorectomized BRCA1/2 mutation carriers. Menopause. 2014 Jul;21(7):763-8

26 International Menopause Society (IMS). Updated 2013 International Menopause Society recommendations on menopausal hormone therapy and preventive strategies for midlife health. Climacteric 2013;16:316-37

27 Von Schoultz E, Rutqvist LE; Stockholm Breast Cancer Study Group. Menopausal hormone replacement therapy after breast cancer: the Stockholm randomised trial. J Natl Cancer Inst 2005;97:533-5

28 Holmberg L, Anderson H. For the HABITS steering and data monitoring committees. HABITS (hormonal replacement therapy after breast cancer - is it safe?), a randomised comparison: trial stopped. Lancet 2004, 363: 453-5

29 Holmberg L, Iversen OE, Rudenstam CM, et al. Increased risk of recurrence after hormone replacement therapy in breast cancer survivors. J Natl Cancer Inst 2008;100:475-82

30 Fahlén M, Fornander T, Johansson H, et al. Hormone replacement therapy after breast cancer: 10 year follow up of the Stockholm randomised trial. Eur J Cancer 2013;49(1):52-9

31 Kenemans P, Bundred NJ, Foidart JM, et al. Safety and efficacy of tibolone in breast-cancer patients with vasomotor symptoms: a doubleblind, randomised, non-inferiority trial. Lancet Oncol 2009;10:135-46

32 Annals of Surgical Oncology 14(9):2425-2427 DOI: 10.1245/s10434-007-9447-z Society of Surgical Oncology: Position Statement on Prophylactic Mastectomy. Approved by the Society of Surgical Oncology Executive Council, March 2007 Armando E. Giuliano, MD, 1 Susan Boolbol, MD, 2 Amy Degnim, MD, 3 Henry Kuerer, MD, 4 A. Marilyn Leitch, MD, 5 and Monica Morrow, MD6 